\title{
QCD axion window and low-scale inflation
}

\author{
Fuminobu Takahashi, ${ }^{1,2,3}$ Wen Yin, ${ }^{4 *}$ and Alan H. Guth ${ }^{3}$ \\ ${ }^{1}$ Department of Physics, Tohoku University, Sendai, Miyagi 980-8578, Japan \\ ${ }^{2}$ Kavli Institute for the Physics and Mathematics of the Universe (WPI), \\ University of Tokyo, Kashiwa 277-8583, Japan \\ ${ }^{3}$ Center for Theoretical Physics and Department of Physics, Massachusetts Institute of Technology, \\ Cambridge, Massachusetts 02139, USA \\ ${ }^{4}$ Institute of High Energy Physics, Chinese Academy of Sciences, Beijing 100049, China
}

(Received 17 June 2018; published 31 July 2018)

\begin{abstract}
We show that the upper bound of the classical QCD axion window can be significantly relaxed for low-scale inflation. If the Gibbons-Hawking temperature during inflation is lower than the QCD scale, the initial QCD axion misalignment angle follows the Bunch-Davies distribution. The distribution is peaked at the strong $C P$ conserving minimum if there is no other light degree of freedom contributing to the strong $C P$ phase. As a result, the axion overproduction problem is significantly relaxed even for an axion decay constant larger than $10^{12} \mathrm{GeV}$. We also provide concrete hilltop inflation models where the Hubble parameter during inflation is comparable to or much smaller than the QCD scale, with successful reheating taking place via perturbative decays or dissipation processes.
\end{abstract}

DOI: 10.1103/PhysRevD.98.015042

\section{INTRODUCTION}

The QCD axion is a pseudo Nambu-Goldstone boson associated with the spontaneous breakdown of a global Peccei-Quinn symmetry [1-3]. Interestingly, the QCD axion not only solves the strong $C P$ problem, but also explains dark matter as it is copiously produced by the initial misalignment mechanism in the early Universe [4-6].

The axion is massless at high temperatures, but it acquires a mass from nonperturbative effects of QCD at low energy, solving the strong $C P$ problem. In the early Universe, therefore, there is no reason for the QCD axion to sit exactly at the $C P$ conserving minimum, and it is usually assumed that the initial position, $a_{*} / f_{a}$, is of order of unity, where $f_{a}$ is the axion decay constant. Then, the QCD axion starts to oscillate about the $C P$ conserving minimum around the QCD phase transition, and the coherently oscillating axion becomes dark matter. See e.g., [7-11] for recent reviews.

The so-called classical axion window is given by

$$
4 \times 10^{8} \mathrm{GeV} \lesssim f_{a} \lesssim 10^{12} \mathrm{GeV},
$$

where the upper bound is due to the axion abundance described above [4-6], and the lower is due to the neutrino burst duration of SN1987A [12-14]. Therefore, if the axion

\footnotetext{
*wyin@ihep.ac.cn
}

Published by the American Physical Society under the terms of the Creative Commons Attribution 4.0 International license. Further distribution of this work must maintain attribution to the author(s) and the published article's title, journal citation, and DOI. Funded by SCOAP ${ }^{3}$. decay constant is of order the GUT scale or string scale, $f_{a} \sim 10^{16-17} \mathrm{GeV}$, the axion abundance exceeds the observed dark matter abundance unless the initial position $a_{*} / f_{a}$ is fine-tuned to be much smaller than unity.

There are several ways to relax the upper bound of the classical axion window. For instance, the axion abundance can be diluted by the late-time entropy production after the QCD phase transition [5,15-18]. A small value of the initial misalignment angle may be selected in the multiverse based on the anthropic principle [19-21]. Alternatively, the axion can acquire a time-dependent mass through the Witten effect, if it is coupled to hidden monopoles [22,23]. If the effect is sizable, the axion follows the time-dependent minimum adiabatically, and no particle production takes place at the time of the QCD phase transition, suppressing the final abundance. Also, if there is a resonant mixing with axionlike particles, the axion abundance can be suppressed by the mass ratio [24-26]. More recently, it was pointed out that, if the axion has a large coupling to massless hidden photons, the axion energy density is dissipated to hidden photons through tachyonic resonance, and the abundance can be suppressed by $\mathcal{O}(100)$ [27] (see also Ref. [28]).

In this paper we propose another simple way to relax the upper bound of the axion window. We show that, if the Hubble parameter during inflation is comparable to or lower than the QCD scale, the axion already acquires a nonzero mass during inflation, and the distribution of its initial position follows the Bunch-Davies distribution [29]. The distribution is peaked at the $C P$ conserving minimum if there is no other light degree of freedom contributing to 
the strong $C P$ phase. In other words, the typical initial misalignment angle is actually given by a function of the Hubble parameter during inflation. As a result, the upper bound of the axion window can be significantly relaxed for such a low-scale inflation. We also provide concrete lowscale inflation models where successful reheating takes place via perturbative decays or dissipation processes.

\section{BUNCH-DAVIES DISTRIBUTION}

In this section we briefly review the Bunch-Davis distribution of a light scalar field $\phi$ during a de Sitter universe or eternal inflation with the Hubble parameter $H_{\text {inf }}$. The action is given by

$$
S=\int d^{4} x \sqrt{-g}\left(-\frac{1}{2} g^{\mu \nu} \frac{\partial \phi}{\partial x^{\mu}} \frac{\partial \phi}{\partial x^{\nu}}-V(\phi)\right) .
$$

For simplicity we assume that the potential for the scalar is given by

$$
V(\phi) \simeq \frac{m_{\phi}^{2}}{2} \phi^{2}+V_{0}
$$

where $V_{0}$ is the positive energy density leading to an exponential expansion of the Universe, and $m_{\phi}(>0)$ is the mass of the scalar. Note that $\phi$ is not the inflaton. Here we neglect the time dependence of $V_{0}$, which would decrease slowly in the case of the usual slow-roll inflation. The Hubble parameter is given by

$$
H_{\mathrm{inf}} \equiv \sqrt{\frac{V_{0}}{3 M_{\mathrm{pl}}^{2}}}
$$

where $M_{\mathrm{pl}} \simeq 2.4 \times 10^{18} \mathrm{GeV}$ is the reduced Planck mass. We assume that the scalar mass is lighter than the Hubble parameter,

$$
m_{\phi} \ll H_{\text {inf }} .
$$

We express the scalar field as $\phi(\mathbf{x}, t)=\delta \phi(\mathbf{x}, t)+\phi_{0}(t)$, and expand the space-dependent part in the Fourier expansion as

$\delta \phi(\mathbf{x}, t)=\int \frac{d^{3} \mathbf{k}}{(2 \pi)^{\frac{3}{2}}}\left[\delta \phi_{k}(t) \alpha_{\mathbf{k}} e^{i \mathbf{k} \cdot \mathbf{x}}+\delta \phi_{k}^{*}(t) \alpha_{\mathbf{k}}^{\dagger} e^{-i \mathbf{k} \cdot \mathbf{x}}\right]$,

where $k=|\mathbf{k}|$, and $\alpha_{\mathbf{k}}$ and $\alpha_{\mathbf{k}}^{\dagger}$ will be later identified with the annihilation and creation operators, respectively. By solving the classical equation of motion for $\phi_{0}$, one can see that $\phi_{0}$ exponentially approaches zero as inflation ends

$$
\phi_{0}\left(t_{f}\right) \simeq e^{-N \frac{m_{\phi}^{2}}{3 H_{\mathrm{inf}}^{2}}} \phi_{0}\left(t_{i}\right),
$$

where $N$ is the $e$-folding number for inflation, and $t_{i}$ and $t_{f}$ are the initial and final cosmic time during inflation. Assuming $N m_{\phi}^{2} / 3 H_{\text {inf }}^{2} \gg 1, \phi_{0}\left(t_{f}\right)$ asymptotes to zero. On the other hand, as we will see below, the quantum fluctuation $\delta \phi$ will be accumulated during inflation, which will dominate over $\phi_{0}$. Therefore we will simply drop the zero mode by setting $\phi_{0}=0$ in the following analysis.

The equation of motion for $\delta \phi_{k}$ reads

$$
\delta \ddot{\phi}_{k}+3 H \delta \dot{\phi}_{k}+\left[m_{\phi}^{2}+\left(\frac{k}{a}\right)^{2}\right] \delta \phi_{k}=0,
$$

where $a(t)=a_{0} e^{H_{\text {inf }} t}$ is the scale factor. It is convenient to use the conformal time defined by

$$
\eta \equiv \int \frac{d t}{a(t)}=-\frac{1}{a H}+C,
$$

where $C$ is a constant. We take hereafter $C=0$ so that

$$
\eta \rightarrow\left\{\begin{array}{ll}
-\infty & \text { as } t \rightarrow-\infty \\
0 & \text { as } t \rightarrow \infty
\end{array} .\right.
$$

The equation of motion can be rewritten as

$$
\frac{1}{k^{2}} \frac{d^{2} X_{k}}{d \eta^{2}}+\frac{1}{k \eta} \frac{1}{k} \frac{d X_{k}}{d \eta}+\left[1-\left(\frac{9}{4}-\frac{m_{\phi}^{2}}{H_{\mathrm{inf}}^{2}}\right) \frac{1}{(k \eta)^{2}}\right] X_{k}=0,
$$

where we have defined $X_{k} \equiv(-\eta)^{-3 / 2} \delta \phi_{k}$. Using the Hankel function, one can express the solution as

$$
X_{k} \propto H_{\nu}^{(1)}(-k \eta)
$$

with $\nu \equiv \sqrt{\frac{9}{4}-\frac{m_{\phi}^{2}}{H_{\mathrm{inf}}^{2}}}$. Then the mode function is given by

$$
\delta \phi_{k}=\frac{\sqrt{\pi}}{2} H_{\mathrm{inf}}(-\eta)^{3 / 2} H_{\nu}^{(1)}(-k \eta)
$$

Here we have adopted the normalization so that it matches with the flat-space time result in the subhorizon limit, $k \eta \rightarrow-\infty$. Since the scalar mass is much smaller than the Hubble parameter, one arrives at

$$
\left|\delta \phi_{k}(t)\right|^{2} \simeq \frac{H_{\mathrm{inf}}^{2}}{2 k^{3}}\left(\frac{k}{a(t) H_{\mathrm{inf}}}\right)^{\frac{2 m_{\phi}^{2}}{3 H_{\mathrm{inf}}^{2}}}
$$

on superhorizon scales, keeping only the leading order of $m_{\phi}^{2} / H_{\mathrm{inf}}^{2}$.

At the subhorizon scales, the scalar field can be quantized in a usual way. Well inside the Hubble horizon, one can neglect the effect of the gravity and quantize the 
scalar field as in the flat spacetime based on the canonical quantization conditions. They are equivalent to imposing the following conditions on the annihilation/creation operators:

$$
\begin{gathered}
{\left[\alpha_{\mathbf{k}}, \alpha_{\mathbf{k}^{\prime}}\right]=0,} \\
{\left[\alpha_{\mathbf{k}}, \alpha_{\mathbf{k}^{\prime}}^{\dagger}\right]=\delta^{(3)}\left(\mathbf{k}-\mathbf{k}^{\prime}\right) .}
\end{gathered}
$$

Then, one can define the Bunch-Davies vacuum by $\alpha_{\mathbf{k}}|0\rangle=0$ for any $\mathbf{k}$ with $\langle 0 \mid 0\rangle=1$ [29]. At the end of inflation $\left(t=t_{f}\right)$, the fluctuations of the scalar field on scales of order the horizon obey a Gaussian distribution, with a variance $\left\langle\phi^{2}\left(t_{f}\right)\right\rangle$ given by

$$
\begin{aligned}
\left\langle\phi^{2}\left(t_{f}\right)\right\rangle & \simeq \int_{a\left(t_{i}\right) H_{\mathrm{inf}}}^{a\left(t_{f}\right) H_{\mathrm{inf}}} \frac{d^{3} \mathbf{k}}{(2 \pi)^{3}}\left|\delta \phi_{k}\left(t_{f}\right)\right|^{2} \\
& =\frac{3 H_{\mathrm{inf}}^{4}}{8 \pi^{2} m_{\phi}^{2}}\left[1-\left(\frac{a\left(t_{i}\right)}{a\left(t_{f}\right)}\right)^{\frac{2 m_{\phi}^{2}}{3 H_{\mathrm{inf}}^{2}}}\right] \\
& \simeq\left(\sqrt{\frac{3}{8 \pi^{2}}} \frac{H_{\mathrm{inf}}^{2}}{m_{\phi}}\right)^{2} .
\end{aligned}
$$

The integration is over the modes that exited the horizon during the period of inflation, from time $t_{i}$ [when $k / a\left(t_{i}\right)=H_{\text {inf }}$ ] to $t_{f}$ [when $k / a\left(t_{f}\right)=H_{\text {inf }}$ ]. The final approximation is justified by the assumption that

$$
\left(\frac{a\left(t_{i}\right)}{a\left(t_{f}\right)}\right)^{\frac{2 m_{\phi}^{2}}{3 H_{\mathrm{inf}}^{2}}}=\exp \left(-\frac{2 m_{\phi}^{2}}{3 H_{\mathrm{inf}}^{2}} N\right) \ll 1,
$$

an assumption that we also made in discussing the behavior of $\phi_{0}(t)$. This approximation is equivalent to setting the lower limit of integration in Eq. (17) to zero.

If we focus on the dynamics of the scalar field after the horizon exit of the CMB scales, the typical initial value of $\phi$ is given by

$$
\phi_{i} \approx \phi_{\mathrm{rms}}=\sqrt{\frac{3}{8 \pi^{2}}} \frac{H_{\mathrm{inf}}^{2}}{m_{\phi}} .
$$

If we allow the initial field value to be fine-tuned, with a probability of $10 \%, 1 \%$, or $0.1 \%$, then $\phi_{i}$ would be bounded by $\left|\phi_{i}\right|<\epsilon \phi_{\mathrm{rms}}$, with

$$
\epsilon \simeq\left\{\begin{array}{ll}
0.126 & (10 \% \text { tuning }) \\
0.0125 & (1 \% \text { tuning }) \\
0.00125 & (0.1 \% \text { tuning })
\end{array} .\right.
$$

In summary, if the inflation lasts sufficiently long, the distribution of the light scalar field is given by a function of the Hubble parameter during inflation and its mass. Even if the mass is lighter than the Hubble parameter, the scalar field knows where the potential minimum is in a probabilistic way. This will be essential when we apply this result to the QCD axion in the next section.

\section{THE QCD AXION ABUNDANCE}

Now let us turn to the QCD axion abundance in a very low-scale inflation. We identify the scalar field $\phi$ in the previous section with the QCD axion, $a$. If the amplitude of $a$ is (much) smaller than $\pi f_{a}$, the previous argument holds without any change.

The strong gauge coupling becomes large and perturbative $\mathrm{QCD}$ breaks down at the QCD scale, $\Lambda_{\mathrm{QCD}}$. The QCD axion acquires a nonzero mass from nonperturbative effects of QCD, and its mass is related to the topological susceptibility $\chi(T)$ as

$$
m_{a}^{2}(T)=\frac{\chi(T)}{f_{a}^{2}}
$$

The temperature dependence of $\chi(T)$ was estimated by several groups using lattice QCD [30-35], and the axion mass is parametrized as

$m_{a}(T) \simeq\left\{\begin{array}{ll}\frac{\sqrt{x_{0}}}{f_{a}}\left(\frac{T_{\mathrm{QCD}}}{T}\right)^{n} & T \gtrsim T_{\mathrm{QCD}} \\ 5.7 \times 10^{-6}\left(\frac{10^{12} \mathrm{GeV}}{f_{a}}\right) \mathrm{eV} & T \lesssim T_{\mathrm{QCD}}\end{array}\right.$,

where the exponent is given by $n \simeq 4.08$ [33], and we adopt $T_{\mathrm{QCD}} \simeq 153 \mathrm{MeV}$ and $\chi_{0} \simeq(75.6 \mathrm{MeV})^{4}$.

The QCD axion starts to oscillate around the minimum when its mass becomes comparable to the Hubble parameter $H$. The axion abundance is given by [36]

$$
\begin{aligned}
\Omega_{a} h^{2} \simeq & 0.35\left(\frac{\theta_{*}}{0.001}\right)^{2} \\
& \times \begin{cases}\left(\frac{f_{a}}{3 \times 10^{17} \mathrm{GeV}}\right)^{1.17} & f_{a} \lesssim 3 \times 10^{17} \mathrm{GeV} \\
\left(\frac{f_{a}}{3 \times 10^{17} \mathrm{GeV}}\right)^{1.54} & f_{a} \gtrsim 3 \times 10^{17} \mathrm{GeV}\end{cases}
\end{aligned}
$$

where the initial misalignment angle is defined by $\theta_{*} \equiv a_{*} / f_{a}$. For $\theta_{*}=1$, one finds that $f_{a}$ should be less than about $10^{12} \mathrm{GeV}$ to avoid the overabundance of the axion dark matter, $\Omega_{a} h^{2} \lesssim 0.12$ [37]. This is nothing but the upper bound of the ordinary axion window (1). In deriving this upper bound, $H_{\text {inf }} \gg \Lambda_{\mathrm{QCD}}$ is implicitly assumed.

If $H_{\text {inf }} \lesssim \Lambda_{\mathrm{QCD}}$, on the other hand, the axion acquires its mass during inflation. ${ }^{1}$ If the inflation lasted sufficiently

\footnotetext{
${ }^{1}$ When $H_{\mathrm{inf}} \sim \Lambda_{\mathrm{QCD}}$, the axion mass is expected to be modified from Eq. (22) due to the difference between the de Sitter and flat space. However, this does not change the following argument significantly.
} 
long (as in the case of eternal inflation), the distribution of the axion field value follows the Bunch-Davies distribution. Thus, the initial misalignment angle is bounded by

$$
\left|\theta_{*}\right| \lesssim \epsilon \sqrt{\frac{3}{8 \pi^{2}}} \frac{H_{\mathrm{inf}}^{2}}{f_{a} m_{a}\left(T_{\mathrm{inf}}\right)},
$$

where

$$
T_{\mathrm{inf}}=\frac{H_{\mathrm{inf}}}{2 \pi}
$$

is the Gibbons-Hawking temperature during the inflation [38], and we have included the fine-tuning parameter, $\epsilon$. It implies that, if $H_{\mathrm{inf}}$ is much smaller than $\Lambda_{\mathrm{QCD}}, \theta_{*}$ becomes much smaller than $\mathcal{O}(1)$,

$$
\left|\theta_{*}\right| \lesssim 0.0034 \epsilon\left(\frac{H_{\mathrm{inf}}}{10 \mathrm{MeV}}\right)^{2} \text { for } T_{\mathrm{inf}} \lesssim T_{\mathrm{QCD}}
$$

Thus, the axion abundance is suppressed in the low-scale inflation, which relaxes the upper bound on the axion window.

Note that we have assumed in the above argument that the strong $C P$ phase during inflation is same as in the current vacuum. This is not an unreasonable assumption because all the particles heavier than $H_{\text {inf }}$ can be integrated out. In particular, the Higgs or the other heavy moduli are stabilized at their minima during inflation. However, if there is another light axionlike particle coupled to gluons, or if the inflaton itself is coupled to gluons, such an assumption does not necessarily hold.

We have numerically solved the equation of motion for the QCD axion field to estimate the axion abundance as a function of the Hubble parameter during inflation. Our result is shown in Fig. 1. The regions above the lines are excluded because the axion is overproduced for the finetuning parameter, $\epsilon=1,0.126,0.0125$, and 0.00125 from left to right. The vertical lines correspond to the upper bounds of the ordinary axion window for $\theta_{*}=1,0.1,0.01$, and 0.001 from left to right. One can see that even $f_{a} \sim$ $10^{18} \mathrm{GeV}$ is allowed and the QCD axion can explain dark matter or a fraction of dark matter for low-scale inflation with $H_{\text {inf }}<\mathcal{O}(1) \mathrm{MeV}$ without any fine-tuning of initial angle. ${ }^{2}$ This is the main result of this paper.

Before closing this section, let us comment on the constraint of isocurvature perturbation. The quantum fluctuation of $a$ would lead to an isocurvature purturbation. The isocurvature power spectrum $\mathcal{P}_{S}$ is constrained to be [42]

$$
\mathcal{P}_{S}<\mathcal{P}_{S}^{\text {bound }} \simeq 8.8 \times 10^{-11},
$$

\footnotetext{
${ }^{2}$ Note that $6 \times 10^{-13} \mathrm{eV} \lesssim m_{a} \lesssim 10^{-11} \mathrm{eV}$, i.e., $6 \times 10^{17} \mathrm{GeV} \lesssim$ $f_{a} \lesssim 10^{19} \mathrm{GeV}$, is disfavored by null observations of the black hole superradiance effect [39-41].
}

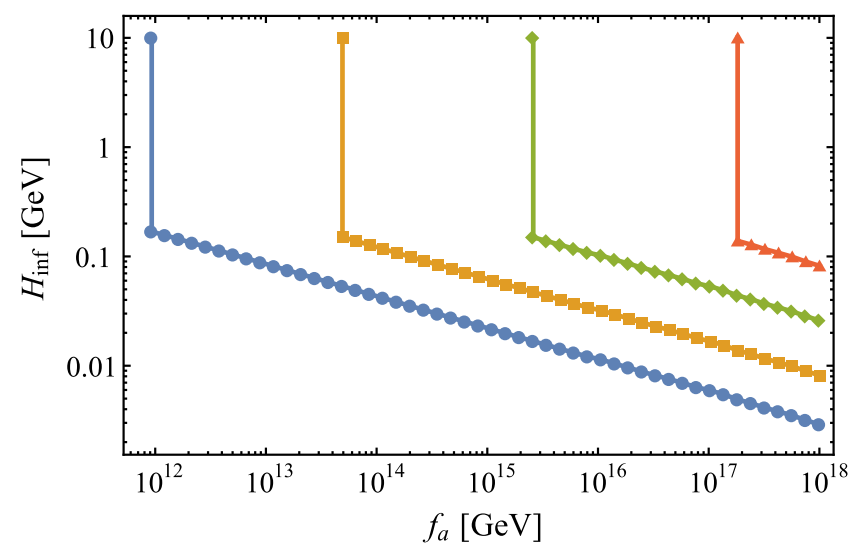

FIG. 1. The relaxed QCD axion window as a function of the inflation scale. Numerical results are shown by symbols for the fine-tuning parameter, $\epsilon=1,0.126,0.0125$, and 0.00125 from left to right. The vertical lines correspond to the upper bounds of the ordinary axion window for $\theta_{*}=1,0.1,0.01$, and 0.001 from left to right. The region above each line is excluded because the axion abundance exceeds the observed dark matter abundance.

at the pivot scale $k_{p}=0.05 \mathrm{Mpc}^{-1}$. This sets an upper bound on the inflation scale as $H_{\text {inf }} \lesssim \mathcal{O}\left(10^{7}\right) \mathrm{GeV}$ for $f_{a}=10^{12} \mathrm{GeV}$. (See Ref. [43] for the anharmonic effect on the isocurvature perturbation.) Since the Hubble parameter of our interest is of order the QCD scale or lower, the isocurvature bound is satisfied.

\section{LOW-SCALE INFLATION WITH $H_{\text {inf }} \lesssim \Lambda_{\text {QCD }}$}

Here we provide concrete low-scale inflation models with $H_{\text {inf }} \lesssim \Lambda_{\mathrm{QCD}}$. In such low-scale inflation models, care must be taken to achieve successful reheating. In particular, if the reheating temperature is lower than the weak scale, then baryogenesis becomes difficult, although not impossible. Also, if one introduces relatively strong couplings of the inflaton to the standard model particles for successful reheating, they may spoil the flatness of the inflaton potential.

We consider a hilltop inflation model with a polynomial potential, where the inflaton $\varphi$ respects a $Z_{2}$ symmetry, following Ref. [44]. The potential has the following form:

$$
V_{\mathrm{inf}}(\varphi)=V_{0}-\frac{m_{0}^{2}}{2} \varphi^{2}-\frac{\kappa}{2 n} \frac{\varphi^{2 n}}{M^{2 n-4}}+\frac{\lambda}{2 m} \frac{\varphi^{2 m}}{M^{2 m-4}},
$$

where $M$ is a cutoff scale, and $m$ and $n$ are integers satisfying $m>n$. For simplicity, let us take $m_{0} \simeq 0$, and we will return to the case of $m_{0} \neq 0$ later. The last term stabilizes the inflaton at

$$
\varphi=\varphi_{\min }=\left(\frac{\kappa}{\lambda}\right)^{\frac{1}{2(m-n)}} M .
$$

The vanishingly small cosmological constant in the present vacuum implies 


$$
\begin{aligned}
V_{0} & =\left(\frac{m-n}{2 m n}\right)\left(\frac{\kappa^{m}}{\lambda^{n}}\right)^{\frac{1}{m-n}} M^{4} \\
& =\left(\frac{m-n}{2 m n}\right) \kappa \varphi_{\min }^{2 n} M^{4-2 n}
\end{aligned}
$$

The inflation occurs in the vicinity of the origin where the inflaton potential is very flat. Inflation ends when one of the slow roll parameters, $\eta \equiv M_{\mathrm{pl}}^{2} V_{\text {inf }}^{\prime \prime} / V_{\text {inf }}$, becomes equal to (minus) unity, ${ }^{3}$ where the prime denotes the derivative with respect to $\varphi$. Solving $\eta=-1$, one obtains

$$
\varphi=\varphi_{\mathrm{end}} \simeq\left[\frac{m-n}{2 m n} \frac{1}{2 n-1}\right]^{\frac{1}{2(n-1)}} \varphi_{\mathrm{min}}^{\frac{n}{n-1}} M_{\mathrm{pl}}^{-\frac{1}{n-1}},
$$

where we used the fact that in these models, $\varphi_{\text {end }} \ll M$. The $e$-folding number is calculated as

$$
\begin{aligned}
N_{*} & \simeq-\int_{\varphi_{*}}^{\varphi_{\mathrm{end}}} d \varphi \frac{3 H_{\mathrm{inf}}^{2}}{V_{\mathrm{inf}}^{\prime}} \simeq \frac{V_{0} M^{2 n-4}}{\kappa M_{\mathrm{pl}}^{2} 2(n-1)} \frac{1}{\varphi_{*}^{2(n-1)}} \\
& \simeq-\frac{2 n-1}{2 n-2} \eta_{*}^{-1} .
\end{aligned}
$$

Here and in what follows the subscript $*$ denotes the value evaluated at horizon exit of the CMB scales; in the second equality we have assumed $\varphi_{*}^{2(n-1)} \ll \varphi_{\text {end }}^{2(n-1)} ; N_{*}$ is the $e$-folding number during the slow-roll from the field value $\varphi_{*}$ to $\varphi_{\text {end }}$ (Notice that $N_{*}$ is smaller than $N$ in Sec. II). The spectral index is given by

$$
n_{s} \simeq 1+2 \eta_{*} \simeq 1-\left(\frac{2 n-1}{n-1}\right) \frac{1}{N_{*}} .
$$

When $n=2$ the inflation model is reduced to the quartic hilltop inflation. In this case the spectral index is known to be too small to be consistent with Planck data for $N_{*} \lesssim 40$ [42]. ${ }^{4}$ The spectral index can be increased by including e.g., a small $Z_{2}$ breaking linear term [45], or a ColemanWeinberg potential [46,47]. As emphasized in Ref. [45], such corrections to the inflaton potential slightly changes the inflaton dynamics in such a way that the inflaton field value at the horizon exit of the CMB scale becomes closer to the origin where $\left|\eta_{*}\right|$ is smaller. To this end, the inflaton mass term $m_{0}$ is not so effective, because it not only changes the inflaton dynamics, but also contributes to $\eta$ in the wrong direction, and these two effects are more or less canceled. In any case, one can easily increase the spectral index to give a better fit to the Planck data, and the inclusion of these effects do not alter the following argument on the reheating.

\footnotetext{
${ }^{3}$ The slow-roll parameter $\eta$ should not be confused with the conformal time in Sec. II.

${ }^{4}$ The typical value of $N_{*}$ is about 40 for the low-scale inflation with $H_{\text {inf }} \sim \Lambda_{\mathrm{QCD}}$.
}

The CMB normalization condition is given by $[42,48]$

$$
\left.A_{s} \equiv \Delta_{\mathcal{R}}^{2} \simeq \frac{V_{\mathrm{inf}}^{3}}{12 \pi^{2}\left(V_{\mathrm{inf}}^{\prime}\right)^{2} M_{\mathrm{pl}}^{6}}\right|_{\varphi=\varphi_{*}} \simeq 2.2 \times 10^{-9} .
$$

This fixes $\kappa$ as

$$
\begin{aligned}
\kappa \simeq & 2.6 \times 10^{-7}\left(\frac{1}{2(n-1) N_{*}}\right)^{\frac{2 n-1}{n-1}}\left(\frac{2 m n}{m-n}\right)^{\frac{n-2}{n-1}} \\
& \times\left(\frac{\varphi_{\min }}{M}\right)^{-\frac{2 n(n-2)}{n-1}}\left(\frac{M_{\mathrm{pl}}}{M}\right)^{\frac{2(n-2)}{n-1}},
\end{aligned}
$$

which is reduced to

$$
\kappa \simeq 5 \times 10^{-13}\left(\frac{40}{N_{*}}\right)^{3}
$$

for $n=2$, independent of $\varphi_{\min }$ and $M$.

Finally the Hubble parameter during inflation and the inflaton mass at the potential minimum are given by

$$
\begin{aligned}
H_{\mathrm{inf}} \simeq & 2.9 \times 10^{-4}\left(\frac{m-n}{2 m n}\right)^{\frac{1}{2(n-1)}}\left(\frac{1}{2(n-1) N_{*}}\right)^{\frac{2 n-1}{2(n-1)}} \\
& \times\left(\frac{\varphi_{\mathrm{min}}^{n}}{M_{\mathrm{pl}}}\right)^{\frac{1}{n-1}}, \\
m_{\varphi}^{2}= & V^{\prime \prime}\left(\varphi_{\mathrm{min}}\right)=2(m-n) \kappa \frac{\varphi_{\min }^{2(n-1)}}{M^{2(n-2)}} \\
\simeq & 2.6 \times 10^{-7}\left(\frac{(m n)^{n-2}(m-n)}{4\left((n-1) N_{*}\right)^{2 n-1}}\right)^{\frac{1}{n-1}}\left(\frac{\varphi_{\min }}{M_{\mathrm{pl}}}\right)^{\frac{2}{n-1}} M_{\mathrm{pl}}^{2} .
\end{aligned}
$$

Notice that both $H_{\text {inf }}$ and $m_{\varphi}$ depend only on $\varphi_{\min }$. We show $H_{\text {inf }}$ and $m_{\varphi}$ as a function of $\varphi_{\min }$ in the cases of $(n, m)=$ $(2,3)$ and $(2,4)$ in Figs. 2 and 3, respectively. One can see that $H_{\text {inf }}=\mathcal{O}(1-100) \mathrm{MeV}$ and $m_{\varphi}=\mathcal{O}\left(10^{5}-10^{6}\right) \mathrm{GeV}$ are realized for $\varphi_{\min }=\mathcal{O}\left(10^{11}-10^{12}\right) \mathrm{GeV} .^{5}$

Characteristically for low-scale inflation models, the value of the slow-roll parameter $\epsilon \equiv \frac{1}{2} M_{\mathrm{pl}}^{2}\left(V_{\mathrm{inf}}^{\prime} / V_{\mathrm{inf}}\right)^{2}$, and hence the tensor/scalar ratio $r$, are extremely small. In particular

$$
\begin{aligned}
\epsilon & \simeq \frac{H_{\mathrm{inf}}^{2}}{8 \pi^{2} M_{\mathrm{pl}}^{2} \Delta_{\mathcal{R}}^{2}} \simeq 5.8 \times 10^{6}\left(\frac{H_{\mathrm{inf}}}{M_{\mathrm{pl}}}\right)^{2} \\
& \simeq 1.0 \times 10^{-30}\left(\frac{H_{\mathrm{inf}}}{1 \mathrm{GeV}}\right)^{2},
\end{aligned}
$$

which leads immediately to

\footnotetext{
${ }^{5}$ The cutoff scale $M$ is of order $M_{\mathrm{pl}}$ for $\lambda=\mathcal{O}(1)$ with $m=3$.
} 


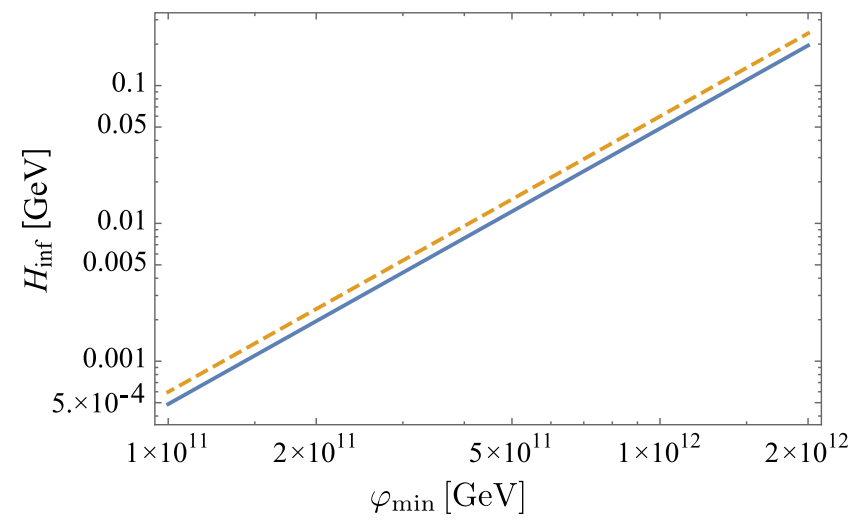

FIG. 2. The Hubble parameter during inflation as a function of the inflaton field value at potential minimum. The blue solid and orange dashed lines correspond to the cases with $(n, m)=(2,3)$ and $(2,4)$, respectively. The $e$-folding number is taken to be $N_{*}=40$.

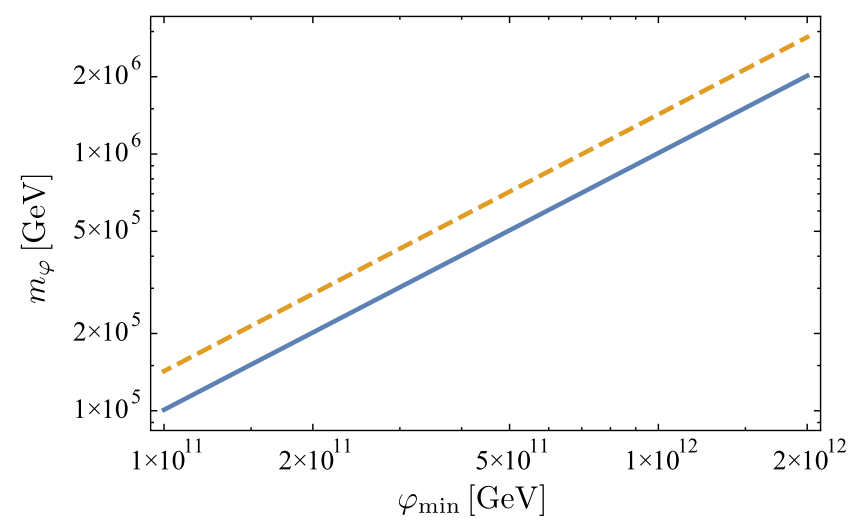

FIG. 3. The inflaton mass as a function of the inflaton field value at a potential minimum. The blue solid and orange dashed lines correspond to the cases with $(n, m)=(2,3)$ and $(2,4)$, respectively. The $e$-folding number is taken to be $N_{*}=40$.

$r \simeq 16 \epsilon \simeq 9.2 \times 10^{7}\left(\frac{H_{\mathrm{inf}}}{M_{\mathrm{pl}}}\right)^{2} \simeq 1.6 \times 10^{-29}\left(\frac{H_{\mathrm{inf}}}{1 \mathrm{GeV}}\right)^{2}$.

For successful reheating we introduce right-handed neutrinos $\nu_{R i}$ which couple to the inflaton with ${ }^{6}$

$$
\sum_{i=1}^{3} \frac{y_{N i}}{\sqrt{2}} \varphi \bar{\nu}_{R i}^{c} \nu_{R i}
$$

Then the inflaton decay rate to a pair of right-handed neutrinos is given by

$$
\Gamma_{\varphi} \simeq \sum_{i}^{N_{\mathrm{R}}^{\mathrm{eff}}} \frac{y_{N i}^{2}}{8 \pi} m_{\varphi},
$$

${ }^{6}$ The inflaton can be identified with the B-L Higgs field. See Refs. $[47,49,50]$ for detailed studies of such inflation model. where the summation is taken over those neutrinos kinematically accessible by the inflaton decay. Let us define the reheating temperature, $T_{R}$, as the temperature at the time $t_{\text {reheat }}$ when the radiation and the inflaton densities are equal. The evolution of the inflaton and radiation can be described by the Boltzmann equations,

$$
\begin{aligned}
& \frac{d \rho_{\varphi}}{d t}=-3 H \rho_{\varphi}-\Gamma_{\varphi} \rho_{\varphi}, \\
& \frac{d \rho_{r}}{d t}=-4 H \rho_{r}+\Gamma_{\varphi} \rho_{\varphi},
\end{aligned}
$$

with the Hubble parameter given by

$$
H=\sqrt{\frac{\rho_{r}+\rho_{\varphi}}{3 M_{\mathrm{pl}}^{2}}},
$$

where $\rho_{\varphi}$ and $\rho_{r}$ are the energy densities of the inflaton and the thermal plasma, respectively. Here we assume that the coherently oscillating inflaton behaves as matter, and the produced right-handed neutrinos immediately decay into the Higgs bosons and leptons. ${ }^{7}$ By solving the above equations numerically, we have found that for $\Gamma_{\varphi} \ll H$, the radiation energy density at $t=t_{\text {reheat }}$ is $0.168 \cdot 3 M_{\mathrm{pl}}^{2} \Gamma_{\varphi}^{2}$, which corresponds to a reheating temperature

$$
\begin{aligned}
T_{R} \simeq & 0.64\left(\frac{90}{\pi^{2} g_{*}}\right)^{\frac{1}{4}} \sqrt{M_{\mathrm{pl}} \Gamma_{\varphi}} \simeq 10 \mathrm{TeV}\left(\frac{106.75}{g_{*}}\right)^{\frac{1}{4}} \\
& \times\left(\frac{y_{N}}{10^{-7}}\right)\left(\frac{m_{\varphi}}{5 \times 10^{5} \mathrm{GeV}}\right)^{\frac{1}{2}}\left(\frac{N_{R}^{\mathrm{eff}}}{2}\right)^{1 / 2},
\end{aligned}
$$

where we have assumed $y_{N i}=y_{N}$ for the kinematically allowed neutrinos. Notice that the right-handed neutrinos are almost massless during inflation due to the $Z_{2}$ symmetry. The right-handed neutrino acquires a mass in the present Universe as

$m_{\nu_{R} i}=\sqrt{2} y_{N i} \varphi_{\min } \simeq 70 \mathrm{TeV}\left(\frac{y_{N i}}{10^{-7}}\right)\left(\frac{\varphi_{\min }}{5 \times 10^{11} \mathrm{GeV}}\right)$.

In this case, the perturbative decay is kinematically allowed and the reheating temperature can be higher than the electroweak scale. This implies that we could have successful baryogenesis via (nonthermal) resonant leptogenesis with $N_{R}^{\text {eff }} \geq 2$ [54-60] or electroweak baryogenesis.

Notice that the inflaton potential receives a radiative correction through the neutrino Yukawa interactions, $\delta V_{\text {inf }} \simeq \frac{y_{N}^{4}}{16 \pi^{2}} \varphi^{4} \log \varphi$, which is negligible compared to the tree-level quartic coupling in the case of $n=2$. However,

\footnotetext{
${ }^{7}$ This assumption is valid around the time of $H \sim \Gamma_{\varphi}$ for the neutrino Yukawa couplings inferred by the seesaw mechanism [51-53] with the light neutrino mass $\simeq 0.05 \mathrm{eV}$.
} 
one can introduce a heavier right-handed neutrino that does not contribute to the decay of the inflaton, but its large Yukawa coupling generates a Coleman-Weinberg potential that increases the spectral index to be consistent with observation [47].

So far, we have discussed low-scale inflation where the reheating proceeds via perturbative decay. When the inflaton couples to standard-model particles strongly enough, the inflaton may dissipate its energy efficiently through scattering with the ambient thermal plasma. This leads to a rather high reheating temperature even for a relatively low inflation scale. In particular, Daido and two of the present authors (FT and WY) recently studied an inflation model where an axionlike particle plays the role of both the inflaton and dark matter $[61,62]$. In this scenario, the reheating proceeds through thermal scatterings with photons and weak gauge bosons, and the inflation scale is extremely low, $H_{\text {inf }}=\mathcal{O}(0.01-1) \mathrm{eV}$. For such an extremely low-scale inflation, the QCD axion abundance is negligible.

\section{DISCUSSION AND CONCLUSIONS}

So far, we have shown that the QCD axion window can be enlarged with low-scale inflation. As a matter of fact, the abundance of axionlike particles can be similarly suppressed. In contrast to the QCD axion, their masses are usually assumed to be independent of time, which makes it much easier to suppress their abundances. In particular, it would be interesting to study cosmology with many light axionlike fields that follow the Bunch-Davies distribution.

In the low-scale model studied above, the inflaton potential respects the $Z_{2}$ symmetry, and so the origin is the symmetry-enhanced point. Therefore, under reasonable assumptions, the inflaton naturally sits at the origin before the last inflation starts. This is the case e.g., if the inflaton acquires a Hubble-induced mass through its coupling to the Ricci curvature during an era of power-law inflation that might precede the final inflation.

We have shown that the upper bound on the axion window can be significantly relaxed in low-scale inflation with the Hubble parameter smaller than the QCD scale. This is because, if the low-scale inflation lasted long enough, the axion initial misalignment angle follows the Bunch-Davies distribution peaked at the strong $C P$ conserving minimum. As a result, the axion overproduction problem is significantly relaxed. We have also provided a concrete low-scale inflation model with successful reheating, where the QCD axion explains dark matter and the baryon asymmetry can be generated via resonant leptogenesis.

\section{ACKNOWLEDGMENTS}

F. T. thanks the hospitality of MIT Center for Theoretical Physics where this work was done. This work is supported by JSPS KAKENHI Grants No. JP15H05889 (F. T.), No. JP15K21733 (F. T.), No. JP17H02878 (F. T.), and No. JP17H02875 (F. T.), Leading Young Researcher Overseas Visit Program at Tohoku University (F. T.), and by World Premier International Research Center Initiative (WPI Initiative), MEXT, Japan (F. T.). A. H. G. is supported in part by the U.S. Department of Energy under grant Contract No. DE-SC0012567.

Note added.-While preparing this paper, we found Ref. [63] which overlaps with the present work. Compared to Ref. [63], we focused more on building a concrete QCD-scale inflation model with successful reheating and pointed out that the absence of any other light scalars (including the inflaton) contributing to the strong $C P$ phase is crucial for the present mechanism to work.
[1] R. D. Peccei and H. R. Quinn, CP Conservation in the Presence of Instantons, Phys. Rev. Lett. 38, 1440 (1977).

[2] R. D. Peccei and H. R. Quinn, Constraints imposed by CP conservation in the presence of instantons, Phys. Rev. D 16, 1791 (1977).

[3] S. Weinberg, A New Light Boson?, Phys. Rev. Lett. 40, 223 (1978).

[4] J. Preskill, M. B. Wise, and F. Wilczek, Cosmology of the invisible axion, Phys. Lett. 120B, 127 (1983).

[5] M. Dine and W. Fischler, The not so harmless axion, Phys. Lett. 120B, 137 (1983).

[6] L. F. Abbott and P. Sikivie, A cosmological bound on the invisible axion, Phys. Lett. 120B, 133 (1983).

[7] J. E. Kim and G. Carosi, Axions and the strong CP problem, Rev. Mod. Phys. 82, 557 (2010).
[8] O. Wantz and E. P. S. Shellard, Axion cosmology revisited, Phys. Rev. D 82, 123508 (2010).

[9] A. Ringwald, Exploring the role of axions and other WISPs in the dark universe, Phys. Dark Universe 1, 116 (2012).

[10] M. Kawasaki and K. Nakayama, Axions: Theory and cosmological role, Annu. Rev. Nucl. Part. Sci. 63, 69 (2013).

[11] D. J. E. Marsh, Axion cosmology, Phys. Rep. 643, 1 (2016).

[12] R. Mayle, J. R. Wilson, J. R. Ellis, K. A. Olive, D. N. Schramm, and G. Steigman, Constraints on axions from SN 1987a, Phys. Lett. B 203, 188 (1988).

[13] G. Raffelt and D. Seckel, Bounds on Exotic Particle Interactions from SN 1987a, Phys. Rev. Lett. 60, 1793 (1988). 
[14] M. S. Turner, Axions from SN 1987a, Phys. Rev. Lett. 60, 1797 (1988).

[15] P. J. Steinhardt and M. S. Turner, Saving the invisible axion, Phys. Lett. 129B, 51 (1983).

[16] G. Lazarides, R. K. Schaefer, D. Seckel, and Q. Shafi, Dilution of cosmological axions by entropy production, Nucl. Phys. B346, 193 (1990).

[17] M. Kawasaki, T. Moroi, and T. Yanagida, Can decaying particles raise the upper bound on the Peccei-Quinn scale?, Phys. Lett. B 383, 313 (1996).

[18] M. Kawasaki and F. Takahashi, Late-time entropy production due to the decay of domain walls, Phys. Lett. B 618, 1 (2005).

[19] A. D. Linde, Axions in inflationary cosmology, Phys. Lett. B 259, 38 (1991).

[20] F. Wilczek, in Universe or Multiverse, edited by B. Carr (Cambridge University Press, Cambridge, England, 2007), p. 151, arXiv:hep-ph/0408167.

[21] M. Tegmark, A. Aguirre, M. Rees, and F. Wilczek, Dimensionless constants, cosmology and other dark matters, Phys. Rev. D 73, 023505 (2006).

[22] M. Kawasaki, F. Takahashi, and M. Yamada, Suppressing the QCD axion abundance by hidden monopoles, Phys. Lett. B 753, 677 (2016).

[23] M. Kawasaki, F. Takahashi, and M. Yamada, Adiabatic suppression of the axion abundance and isocurvature due to coupling to hidden monopoles, J. High Energy Phys. 01 (2018) 053.

[24] N. Kitajima and F. Takahashi, Resonant conversions of QCD axions into hidden axions and suppressed isocurvature perturbations, J. Cosmol. Astropart. Phys. 01 (2015) 032.

[25] R. Daido, N. Kitajima, and F. Takahashi, Domain wall formation from level crossing in the axiverse, Phys. Rev. D 92, 063512 (2015).

[26] R. Daido, N. Kitajima, and F. Takahashi, Level crossing between the QCD axion and an axionlike particle, Phys. Rev. D 93, 075027 (2016).

[27] N. Kitajima, T. Sekiguchi, and F. Takahashi, Cosmological abundance of the QCD axion coupled to hidden photons, Phys. Lett. B 781, 684 (2018).

[28] P. Agrawal, G. Marques-Tavares, and W. Xue, Opening up the QCD axion window, J. High Energy Phys. 03 (2018) 049.

[29] T. S. Bunch and P. C. W. Davies, Quantum field theory in de Sitter space: Renormalization by point splitting, Proc. R. Soc. A 360, 117 (1978).

[30] E. Berkowitz, M. I. Buchoff, and E. Rinaldi, Lattice QCD input for axion cosmology, Phys. Rev. D 92, 034507 (2015).

[31] C. Bonati, M. D’Elia, M. Mariti, G. Martinelli, M. Mesiti, F. Negro, F. Sanfilippo, and G. Villadoro, Axion phenomenology and $\theta$-dependence from $N_{f}=2+1$ lattice QCD, J. High Energy Phys. 03 (2016) 155.

[32] P. Petreczky, H. P. Schadler, and S. Sharma, The topological susceptibility in finite temperature QCD and axion cosmology, Phys. Lett. B 762, 498 (2016).

[33] S. Borsanyi et al., Calculation of the axion mass based on high-temperature lattice quantum chromodynamics, Nature (London) 539, 69 (2016).

[34] J. Frison, R. Kitano, H. Matsufuru, S. Mori, and N. Yamada, Topological susceptibility at high temperature on the lattice, J. High Energy Phys. 09 (2016) 021.
[35] Y. Taniguchi, K. Kanaya, H. Suzuki, and T. Umeda, Topological susceptibility in finite temperature $(2+1)$-flavor QCD using gradient flow, Phys. Rev. D 95, 054502 (2017).

[36] G. Ballesteros, J. Redondo, A. Ringwald, and C. Tamarit, Standard model-axion-seesaw-Higgs portal inflation. Five problems of particle physics and cosmology solved in one stroke, J. Cosmol. Astropart. Phys. 08 (2017) 001.

[37] P. A. R. Ade et al. (Planck Collaboration), Planck 2015 results. XIII. Cosmological parameters, Astron. Astrophys. 594, A13 (2016).

[38] G. W. Gibbons and S. W. Hawking, Cosmological event horizons, thermodynamics, and particle creation, Phys. Rev. D 15, 2738 (1977).

[39] A. Arvanitaki, M. Baryakhtar, and X. Huang, Discovering the QCD axion with black holes and gravitational waves, Phys. Rev. D 91, 084011 (2015).

[40] V. Cardoso, Ó. J. C. Dias, G. S. Hartnett, M. Middleton, P. Pani, and J.E. Santos, Constraining the mass of dark photons and axion-like particles through black-hole superradiance, J. Cosmol. Astropart. Phys. 03 (2018) 043.

[41] M. J. Stott and D. J. E. Marsh, Black hole spin constraints on the mass spectrum and number of axion-like fields, arXiv:1805.02016.

[42] P. A. R. Ade et al. (Planck Collaboration), Planck 2015 results. XX. Constraints on inflation, Astron. Astrophys. 594, A20 (2016).

[43] T. Kobayashi, R. Kurematsu, and F. Takahashi, Isocurvature constraints and anharmonic effects on QCD axion dark matter, J. Cosmol. Astropart. Phys. 09 (2013) 032.

[44] K. Nakayama and F. Takahashi, Low-scale supersymmetry from inflation, J. Cosmol. Astropart. Phys. 10 (2011) 033.

[45] F. Takahashi, New inflation in supergravity after Planck and LHC, Phys. Lett. B 727, 21 (2013).

[46] A. D. Linde, Particle physics and inflationary cosmology (Harwood Academic Publishers, Chur, Switzerland, 1990), arXiv:hep-th/0503203.

[47] K. Nakayama and F. Takahashi, PeV-scale supersymmetry from new inflation, J. Cosmol. Astropart. Phys. 05 (2012) 035 .

[48] H. V. Peiris et al. (WMAP Collaboration), First year Wilkinson microwave anisotropy probe (WMAP) observations: Implications for inflation, Astrophys. J. Suppl. Ser. 148, 213 (2003).

[49] S. F. King and P. O. Ludl, Supersymmetric Majoron inflation, J. High Energy Phys. 03 (2017) 174.

[50] S. Antusch and K. Marschall, Non-thermal leptogenesis after Majoron hilltop inflation, J. Cosmol. Astropart. Phys. 05 (2018) 015.

[51] T. Yanagida, Horizontal symmetry and masses of neutrinos, in Proceedings of the Workshop on the Baryon Number of the Universe and Unified Theories, 1979, edited by O. Sawada and A. Sugamoto (National Lab for High Energy Physics, Tsukuba, Japan, 1979).

[52] M. Gell-Mann, P. Ramond, and R. Slansky, Complex spinors and unified theories, in Proceedings of the Supergravity Workshop, Stony Brook, New York, 1979, edited by P. Van Nieuwenhuizen and D. Z. Freedman (North Holland Publishing Co., Amsterdam, 1979).

[53] P. Minkowski, $\mu \rightarrow e \gamma$ at a rate of one out of $10^{9}$ muon decays?, Phys. Lett. 67B, 421 (1977). 
[54] A. Pilaftsis, Resonant CP violation induced by particle mixing in transition amplitudes, Nucl. Phys. B504, 61 (1997).

[55] W. Buchmuller and M. Plumacher, CP asymmetry in Majorana neutrino decays, Phys. Lett. B 431, 354 (1998).

[56] A. Pilaftsis and T. E. J. Underwood, Resonant leptogenesis, Nucl. Phys. B692, 303 (2004).

[57] A. Pilaftsis and T.E. J. Underwood, Electroweak-scale resonant leptogenesis, Phys. Rev. D 72, 113001 (2005).

[58] A. Anisimov, A. Broncano, and M. Plumacher, The CPasymmetry in resonant leptogenesis, Nucl. Phys. B737, 176 (2006).
[59] M. Garny, A. Kartavtsev, and A. Hohenegger, Leptogenesis from first principles in the resonant regime, Ann. Phys. (Amsterdam) 328, 26 (2013).

[60] B. Dev, M. Garny, J. Klaric, P. Millington, and D. Teresi, Resonant enhancement in leptogenesis, Int. J. Mod. Phys. A 33, 1842003 (2018).

[61] R. Daido, F. Takahashi, and W. Yin, The ALP miracle: unified inflaton and dark matter, J. Cosmol. Astropart. Phys. 05 (2017) 044.

[62] R. Daido, F. Takahashi, and W. Yin, The ALP miracle revisited, J. High Energy Phys. 02 (2018) 104.

[63] P. W. Graham and A. Scherlis, The stochastic axion scenario, arXiv:1805.07362. 